\title{
Efecto de la riqueza de especies y estructura de la vegetación en el almacenamiento de carbono en sistemas agroforestales de la Amazonía, Bolivia
}

\author{
Ernesto Gómez Cardozo ${ }^{1}$, Guillaume Xavier Rousseau ${ }^{1 *}$, Danielle Celentano ${ }^{1}$, \\ Heriberto Fariñas Salazar ${ }^{2} \&$ Christoph Gehring ${ }^{1}$ \\ 1. Programa de Pós-Graduação em Agroecologia, Universidade Estadual do Maranhão, São Luís, MA, Brasil. CEP \\ 65055-970; egomezca@hotmail.com, guilirous@yahoo.ca, danicelentano@yahoo.com.br, \\ christophgehring@yahoo.com.br \\ 2. Centro de Investigación Agrícola Tropical, Av. Irala, Santa Cruz de la Sierra, Bolivia; hfarinas@ciatbo.org \\ * Correspondencia
}

Recibido 31-V-2018. Corregido 27-VII-2018. Aceptado 31-VIII-2018.

\begin{abstract}
Effect of species richness and vegetation structure on carbon storage in agroforestry systems in the Southern Amazon of Bolivia. Diverse agroforestry systems conciliate food production, biodiversity conservation, and the provision of ecosystem services as atmospheric carbon sequestration. However, the role of floristic richness in the production of biomass in these systems is not clear. This study evaluated the effect of species richness and vegetation structure on aboveground biomass carbon in different agroforestry systems in the Southern Amazon of Bolivia. For that, 25 agroforestry systems and 4 secondary forests were studied in the departments of Santa Cruz and Beni. In each system, a $1963 \mathrm{~m}^{2}$ circular plot was installed, where the vegetation (trees, shrubs and herbaceous) and necromass (leaf litter, branches and dead trees) were sampled. Linear and logarithmic functions were used to evaluate the effect of vegetation richness and structure on carbon, and the variance partition was used to examine the pure and shared effect of the richness and vegetation structure variables on carbon. Regressions showed a positive strong relationship between species richness and carbon $\left(\mathrm{r}^{2}=0.74 ; \mathrm{P}<0.001\right)$. The partition of carbon variance showed that richness, structure and variation of the structure explained $85.7 \%$. Alone the richness explained $12.7 \%$, the structure $8.8 \%$ and the variation of the structure $4.8 \%$. These results confirm that carbon in the aboveground biomass increases with species richness and structural variation of the vegetation. Therefore, more biodiverse and stratified agroforestry systems are more efficient in the use of resources and can contribute to climate change mitigation.
\end{abstract}

Key words: biodiversity; biomass; allometric formula; Amazon; climate change mitigation.

Gómez Cardozo, E., Rousseau, G. X., Celentano, D., Fariñas Salazar, H. \& Gehring, C. (2018).

Efecto de la riqueza de especies y estructura de la vegetación en el almacenamiento de carbono en sistemas agroforestales de la Amazonía, Bolivia. Revista de Biología Tropical, 66(4), 1481-1495.

La diversidad de especies en los bosques tropicales es fundamental para su funcionamiento, resiliencia y capacidad de proporcionar bienes y servicios (Cardinale et al., 2011, 2012; Hooper et al., 2012; Gamfeldt et al., 2013). En la Amazonía, la diversidad florística está siendo afectada por la degradación y conversión de bosques naturales en agroecosistemas simples como pastos o monocultivos agrícolas y forestales (Davidson et al., 2012), esta situación es objeto de atención, porque conlleva no solo a la pérdida de biodiversidad, sino también de servicios ecosistémicos esenciales, como el almacenamiento de carbono (Fauset et al., 2015; Poorter et al., 2015). En efecto, los bosques naturales complejos poseen una diversidad de 
especies de plantas con rasgos funcionales y patrones diferentes de crecimiento y tamaño, esto conduce a una estratificación del ecosistema (Poorter et al., 2016), ofreciendo de esta manera mayores oportunidades a las especies para un mejor aprovechamiento de los recursos (luz, agua y nutrientes), debido a la complementariedad de nichos (Loreau, 2000; Díaz \& Cabido, 2001; Coomes et al., 2009). Esta característica, promueve la producción de biomasa en los ecosistemas naturales (Wang et al., 2011; Poorter et al., 2015; Zhang \& Chen, 2015).

Los agroecosistemas biodiversos, que combinan especies leñosas perennes (árboles, palmeras) y cultivos agrícolas como los sistemas agroforestales (SAF) (Nair, 1993; Young, 1997), han ganado gran importancia en los últimos años, porque promueven la biodiversidad y servicios ecosistémicos, además de producir alimentos, generan ingresos económicos y mayor satisfacción a los agricultores (Matocha, Schroth, Hills, \& Hole, 2012; Cardozo et al., 2015; Zomer et al., 2016), también reducen la presión sobre los bosques naturales (Schroth et al., 2015). Existen diferentes prácticas agroforestales (Atangana, Khasa, Chang, \& Degrande, 2014; Nair, 2014), que se practican en más de 1023 millones de hectáreas en todo el mundo (Nair, Kumar, \& Nair, 2009), y en comparación a las prácticas convencionales de ganadería extensiva y agricultura de corte y quema, son una alternativa más sostenible (Yamada \& Gholz, 2002; Leite et al., 2016).

Las prácticas agroforestales tienen el potencial para contribuir a la mitigación del cambio climático, mediante el secuestro del carbono en la vegetación y en el suelo (IPCC, 2000). El potencial de almacenamiento de carbono depende del tipo de práctica agroforestal, composición florística y manejo (Albrecht \& Kandji, 2003; Kumar, 2006; Zomer et al., 2016). La diversidad en la composición florística de los SAF es clave para su funcionamiento y la generación de beneficios (Pinho, Miller, \& Alfaia, 2012). Ali \& Mattsson (2017a) identificaron una relación positiva de la diversidad florística sobre la biomasa en huertos caseros en regiones secas. Sin embargo, existe escasa información sobre esas relaciones para las regiones tropicales. El objetivo de este estudio fue evaluar el efecto de la riqueza de especies y la estructura de la vegetación sobre el almacenamiento de carbono en la biomasa en diferentes sistemas agroforestales en la Amazonía Boliviana. Ese conocimiento es muy relevante para entender el funcionamiento de los sistemas agroforestales, así como para promover políticas públicas para la adopción de sistemas que optimicen la diversidad y el almacenamiento de carbono.

\section{MATERIALES Y MÉTODOS}

Área de estudio: El estudio se realizó en la Amazonía sur de Bolivia, en ocho municipios, en los departamentos de Beni (San Ignacio de Moxos, San Andrés y San Javier) y Santa Cruz (Yapacaní, San Carlos, Buena Vista, Ascensión de Guarayos y Urubichá) (Fig. 1). La clasificación climática de acuerdo con Köppen es tropical Am y Aw (Peel, Finlayson, \& McMahon, 2007), la precipitación anual varía de $1800 \mathrm{~mm}$ en Ascensión de Guarayos y Urubichá a $2800 \mathrm{~mm}$ en Yapacaní, Buena Vista y San Carlos, con cinco a seis meses de estación seca y una temperatura promedio de $25{ }^{\circ} \mathrm{C}$. Los suelos predominantes son de origen aluvial, clasificados como cambisol (Inceptsol), pluvisol (Entisol-Fluvent) y ferrasol (Oxisol) (Quesada et al., 2011, 2012). La vegetación de la región corresponde al bosque amazónico siempre verde, donde los sistemas de uso de suelo predominantes son pastos extensivos, agricultura mecanizada y agricultura de pequeña escala (Müller, Müller, Schierhorn, Gerold, \& Pacheco, 2012).

Sistemas de uso del suelo: En este estudio se evaluaron los sistemas agroforestales (SAF) más representativos de la región. El sistema "Cacao agroforestal" (CA), donde el cacao (Theobroma cacao) es el principal cultivo, y está asociado con otros frutales como los cítricos (Citrus spp.) y bananos (Musa spp.), también incluyen especies maderables como mara (Swietenia macrophylla) y serebo 


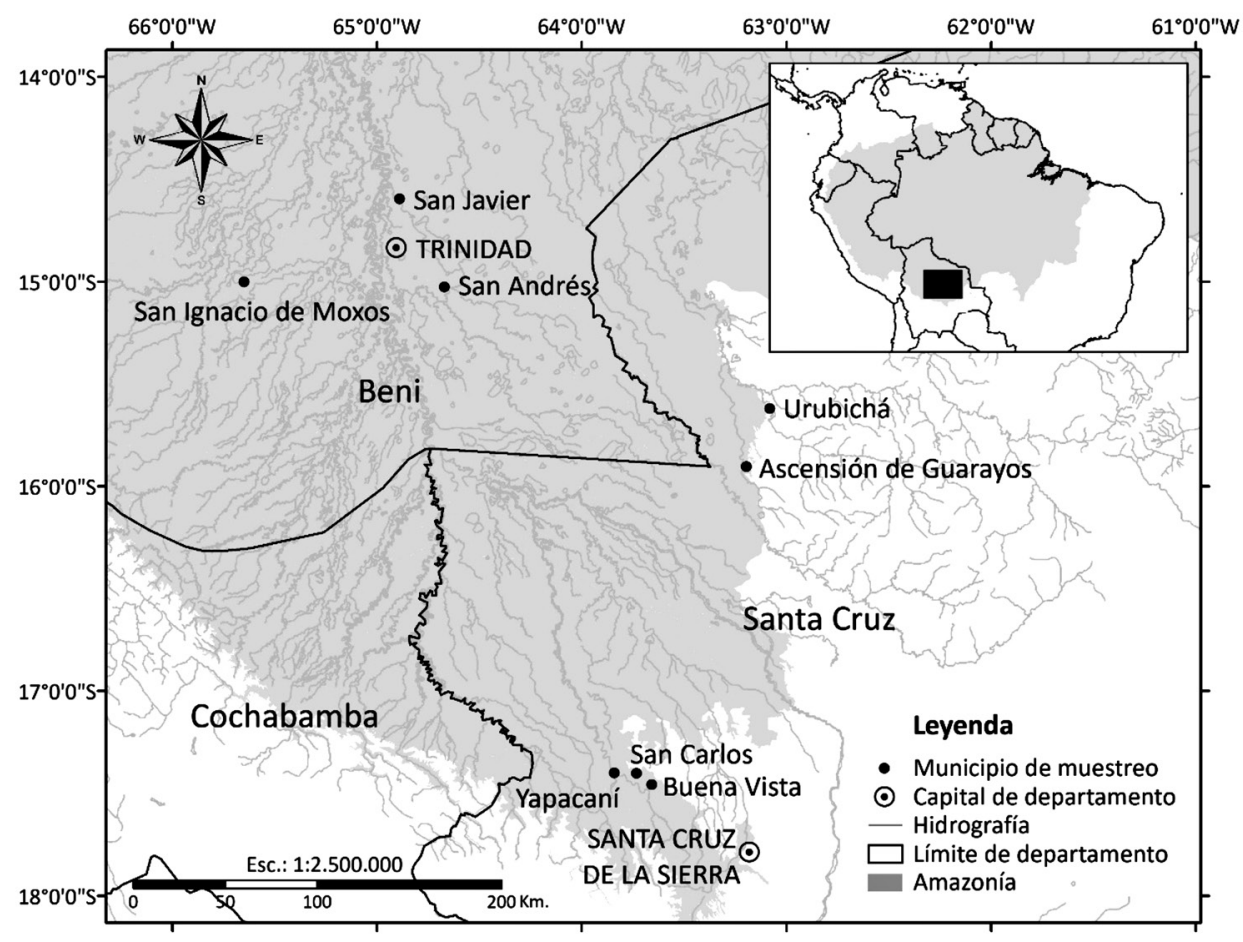

Fig. 1. Áreas de estudio en la Amazonía Sur, Bolivia. / Fig. 1. Study areas in the Southern Amazon, Bolivia.

(Schizolobium parahyba). La mayoría de las especies tienen espacios definidos. El sistema "Café sombreado" (CS), donde el principal cultivo es el café (Coffea arabica), plantado en áreas de bosques secundarios, donde las especies de interés como leguminosas o maderables son seleccionadas y manejadas por el agricultor, ejemplo pacay (Inga sp.), jorori (Swartizia jorori), y las demás especies son cortadas para reducir la sombra. En estos sistemas, una vez al año se realiza la poda para regular la sombra, algunos sistemas han sido enriquecido con especies frutales, achachairu (Garcinia humilis), ocoro (Garcinia madruno) y maderas de valor comercial como almendrillo (Dipteryx odorata), jichituriqui (Aspidosperma macrocarpon).

El sistema "Huerto casero" (HC), uno de los más practicados en los trópicos (Kumar \& Nair, 2004), es caracterizado por estar cerca de la casa, poseen una gran diversidad de frutales, árboles maderables y palmeras, no predomina una especie en particular, es fuente de alimentos y un espacio de descanso para las familias. Además de los sistemas mencionados, "Bosques Secundarios Avanzados" (BS) fueron incluidos en el estudio como ecosistemas de referencia, esos bosques son áreas de regeneración natural en sitios previamente utilizados para la agricultura de corte y quema.

Diseño experimental: Fueron muestreados 25 SAF con diferentes edades (10 sistemas CA de 7 a 40 años, 7 sistemas CS de 8 a 30 años, 8 HC de 14 a 40 años) y 4 BS de 40 a 60 años (Apéndice 1). Las edades de los SAF y BS fueron informadas por los agricultores y la recolecta de los datos fue realizada entre agosto 2015 y diciembre 2016. En cada uno de los 25 SAF y 4 BS estudiados, fue instalada una parcela circular de $50 \mathrm{~m}$ de diámetro $\left(1963 \mathrm{~m}^{2}\right)$, donde se evaluó la biomasa en los diferentes compartimientos: árboles (diámetro a la altura del pecho $\geq 10 \mathrm{~cm}$ ), arbustos (diámetro altura base de 1 a $9 \mathrm{~cm}$ a $30 \mathrm{~cm}$ del suelo y con una altura $\geq 1.5 \mathrm{~m}$ ), necromasa fina (ramas finas 
de 2 a $5 \mathrm{~cm}$ de diámetro), hojarasca (hojas, flores, frutos y ramas $<2 \mathrm{~cm}$ de diámetro), y necromasa gruesa (ramas y árboles caídos $>5$ cm de diámetro).

Método de muestreo de campo: La metodología de recolecta y análisis de la biomasa fue basada en estudios realizados en los trópicos húmedos (Brown, 2002; Chave et al., 2005, 2014; Poorter et al., 2016). Todos los árboles fueron identificados y medidos (diámetro y altura) en las parcelas de $50 \mathrm{~m}$ de diámetro. Para la medición fueron utilizados cinta diamétrica y clinómetro. Los cítricos y el cacao fueron medidos a $30 \mathrm{~cm}$ de la base de suelo (Schroth, D’Angelo, Teixeira, Haag, \& Lieberei, 2002; Andrade, Segura, Somarriba, \& Villalobos, 2008). La identificación de las especies se realizó en campo, con la ayuda del agricultor y de guías dendrológicas (Justiniano et al., 2004; Vargas, Mostacedo, \& Jordán, 2005).

Los arbustos fueron muestreados en cinco sub parcelas de $5 \times 5 \mathrm{~m}\left(25 \mathrm{~m}^{2}\right)$ instaladas dentro de las parcelas circulares, donde se midió el diámetro de todas las especies con un calibrador. Al centro de cada una de estas sub parcelas, se instaló un cuadrante de $1 \mathrm{x} 1 \mathrm{~m}$, donde las especies herbáceas fueron muestreadas de forma destructiva y pesadas en campo. En esos cuadrantes también se muestreo la necromasa fina y la hojarasca que fueron pesadas en el campo para obtener el peso fresco. Finalmente, se recorrió dos transectos de $50 \mathrm{~m}$ en la parcela principal (sentido este - oeste y norte - sur) donde mediante interceptación fue medido el diámetro y estado de descomposición de la necromasa gruesa (Coomes, Allen, Scott, Goulding, \& Beets, 2002; Van Wagner, 1968).

Cálculo de la diversidad, biomasa $\mathbf{y}$ carbono: Como medida de diversidad, se consideró el índice de diversidad de Shannon y la riqueza de especies (número total de especies arbóreas por parcela), este último es de fácil interpretación y también relevante para estudiar los mecanismos de complementariedad de nichos (Loreau et al., 2001; Flombaum \& Sala, 2008; Chisholm et al., 2013)

La biomasa en las especies arbóreas y arbustivas fue calculada con fórmulas alométricas específicas y multiespecíficas, utilizadas en los trópicos húmedos (Cuadro 1). Para las especies arbóreas identificadas fue utilizada la

\section{CUADRO 1}

Ecuaciones alométricas utilizadas para la estimación de la biomasa en los sistemas agroforestales y bosques secundarios en la Amazonía Sur, Bolivia

TABLE 1

Allometric equations used for biomass estimation in agroforestry systems and secondary forests in the Southern Amazon, Bolivia

\begin{tabular}{llll} 
& \multicolumn{1}{c}{ Fórmula } & $\mathrm{R}^{2}$ & \multicolumn{1}{c}{ Fuente } \\
$\begin{array}{ll}\text { Theobroma cacao } \\
\text { Coffea } \text { arabica }\end{array}$ & $\mathrm{BA}=10^{(-1.625+2.63 * \log (\mathrm{D} 30))}$ & 0.98 & (Andrade et al., 2008) \\
Musa spp. & $\mathrm{BA}=10^{(-0.779+2.338 * \log 10(\mathrm{H}))}$ & 0.82 & (Segura, Kanninen, \& Suárez, 2006) \\
Citrus sp. & $\mathrm{BA}=0.030^{*} \mathrm{Dap}^{2.13}$ & 0.99 & (Pearson, Walker, \& Brown, 2005) \\
Cecropia sp. & $\mathrm{BA}=-6.64+0.279 * \mathrm{AB}+0.000514 * \mathrm{AB}^{2}$ & $0.94 \quad$ (Schroth et al., 2002) \\
Palmeras & $\mathrm{BA}=\exp (-2.5118+2.4257 * \ln (\mathrm{Dap}))$ & 0.98 (Nelson et al., 1999) \\
Arbustos $<10$ Dap & $\mathrm{BA}=10.0+6.4 * \mathrm{H}$ & 0.96 (Brown, 1997) \\
Predicción Dap arbustivos & $\mathrm{BA}=\exp (-1.9968+2.4128 \ln (\mathrm{Dap}))$ & 0.98 (Nelson et al., 1999) \\
Árboles de sombra & $\mathrm{Dap}=(-1,038-0,045) * \mathrm{DB}^{2}+1,416 * \mathrm{DB}$ & 0.98 (Gehring, Park, \& Denich, 2008) \\
\hline
\end{tabular}

$\mathrm{BA}=$ Biomasa aérea $(\mathrm{kg}) ; \mathrm{AB}=$ Área basal $\left(\mathrm{m}^{2}\right) ; \mathrm{Dap}=$ Diámetro a la altura del pecho $(\mathrm{cm}) ; \mathrm{D}=$ Diámetro $(\mathrm{cm}) ; \mathrm{H}=\mathrm{Altura}$ $(\mathrm{m}) ; \mathrm{DB}=$ Diámetro basal $(30$ sobre el suelo en $\mathrm{cm}) ; \rho=$ Densidad de la madera $\left(\mathrm{g} / \mathrm{cm}^{3}\right)$.

$\mathrm{BA}=$ Aboveground biomass $(\mathrm{kg}) ; \mathrm{AB}=$ Basal area $\left(\mathrm{m}^{2}\right) ;$ Dap $=$ Diameter at Breath Height $(\mathrm{cm}) ; \mathrm{D}=$ Diameter $(\mathrm{cm}) ; \mathrm{H}=$ Height $(\mathrm{m}) ; \mathrm{DB}=$ Basal diameter (on the floor in $30 \mathrm{~cm}) ; \rho=$ Density of the wood $\left(\mathrm{g} / \mathrm{cm}^{3}\right)$. 
densidad específica de la madera $\left(\mathrm{g} \mathrm{cm}^{3}\right)$, obtenida de la base de datos global (Zanne et al., 2009), para las especies que no están presentes en la lista, se utilizó una densidad media de $0.58 \mathrm{~g} \mathrm{~cm}^{3}$ (Brown, 1997; Nogueira, Fearnside, \& Nelson, 2008). Para la determinación de la biomasa de la vegetación herbácea, necromasa fina y hojarasca, $500 \mathrm{~g}$ de muestras homogenizadas fueron secadas a $65{ }^{\circ} \mathrm{C}$ hasta obtener peso constate para determinar la humedad y luego el porcentaje de masa seca. La biomasa de la necromasa gruesa fue estimada a partir del diámetro y la densidad de la madera (Van Wagner, 1968), que fue clasificada en solida $\left(0.60 \mathrm{~g} \mathrm{~cm}^{3}\right)$, intermedia $\left(0.42 \mathrm{~g} \mathrm{~m}^{3}\right)$ y descompuesta $\left(0.23 \mathrm{~g} \mathrm{~m}^{3}\right)$ (IPCC, 2007).

Para el cálculo del carbono en cada compartimiento (árboles, arbustos, herbáceas, necromasa y hojarasca) la biomasa se multiplicó por el factor 0.5 (IPCC, 2003), luego fue sumada por parcela y extrapolada a $\mathrm{Mg} / \mathrm{ha}$.

Análisis de la información: Inicialmente, fueron evaluadas la normalidad y homogeneidad de la varianza de los datos para cumplir con los supuestos de la estadística paramétrica. Se utilizaron regresiones lineales y logarítmicas para evaluar el efecto de la diversidad, riqueza de especies, variación de la estructura (altura y Dap) y edad de los sistemas agroforestales sobre la acumulación del carbono.

Para determinar la variación de la estructura de la vegetación arbórea (altura y Dap), se calculó el coeficiente de variación $(\mathrm{CV})$ de la altura y Dap, como la proporción de la desviación estándar de todas las mediciones de altura y Dap con la media de la altura y Dap, dentro de cada SAF (Ali \& Mattsson, 2017b). La variación global de la altura y Dap representa el grado de diferenciación de nicho realizado a través de interacciones positivas planta-planta (Clark et al., 2007).

Para examinar como las variables de diversidad (Shannon, riqueza) y estructura (altura, Dap y CV) explican el carbono en la biomasa sobre el suelo (CBS), se utilizó la partición de la varianza. Este examen se basa en el análisis de redundancia (RDA), que examina cuanto de la variación es explicada por un conjunto de variables (Rousseau, Rioux, \& Dostaler, 2006; Borcard, Gillet, Legendre, \& Legendre, 2011). Las variables de diversidad y estructura fueron sometidas a una selección hacia adelante (Forward selection). Este procedimiento seleccionó variables significativas (riqueza, altura, Dap y CV) para construir un modelo que explique la variación del CBS, el índice de Shannon fue excluido del modelo $(\mathrm{P}=0.06)$. Posteriormente la contribución de cada variable en el modelo fue evaluada por el test de Monte Carlo, con 999 permutaciones. Esto permitió especificar cuánto de la variación del modelo final fue explicado por el efecto puro de cada variable y que proporción fue compartida.

Todos los análisis estadísticos se realizaron con el software R 3.3.2. (R Core Team, 2016), la partición de la varianza y RDA utilizó la función varpart en el paquete vegan (Oksanen et al., 2015).

\section{RESULTADOS}

Diversidad: En los 25 sistemas agroforestales (SAF) y 4 bosques secundarios (BS) estudiados fueron encontrados entre árboles, palmeras y bananos (especies arbóreas) un total de 2911 individuos, donde se identificaron 78 especies, distribuidas en 64 géneros y 29 familias. La riqueza del sistema cacao agroforestal (CA) varío entre 4 a 15 especies arbóreas por parcela (promedio $=10.8 \pm 2.9$ ), café sombreado (CS) entre 10 a $18(13.8 \pm 3.4)$, huerto casero (HC) entre 13 a $17(14.8 \pm 1.6)$ y BS entre 18 a $22(20.3 \pm 2.1)$. El índice de diversidad de Shannon del CA varío entre 1.1 a 1.8 por parcela (Promedio $=1.5 \pm 0.07$ ), CS entre 1.7 a $2.3(2.1 \pm 0.08)$, HC entre 1.2 a 2.3 (1.9 $\pm 0.12)$ y BS entre 1.7 a $3.0(2.2 \pm 0.27)$. Las especies más abundantes fueron cacao (Theobroma cacao, $\mathrm{N}=985$ ) que representa el 33.8 $\%$ de las especies arbóreas, seguido por banano (Musa spp., $\mathrm{N}=163,5.6 \%$ ) y motacú (Attalea princeps, $\mathrm{N}=126,4.3 \%$ ). La taxonomía y uso de las 10 especies arbóreas más abundantes se presentan en el apéndice 2 . 
Carbono: El carbono en la biomasa sobre el suelo (CBS) varió entre 14.93 a $68.88 \mathrm{Mg} / \mathrm{ha}$ (Promedio $=32.5 \pm 17$ ) en el CA, 14.43 a 78.58 $(48.8 \pm 22.5)$ en el CS, 30.66 a $55.53(45.6 \pm$ 8.9) en el HC, y 60.22 a $76.51(67.4 \pm 6.9)$ en el BS. Las relaciones bivariadas indicaron que el CBS aumentó significativamente con la riqueza de especies $\left(\mathrm{r}^{2}=0.74 ; \mathrm{P}<0.001\right)$ (Fig. 2a), la edad $\left(\mathrm{r}^{2}=0.61 ; \mathrm{P}<0.001\right)$ (Fig. 2b), la variación de la altura $\left(\mathrm{r}^{2}=0.32 ; \mathrm{P}<0.001\right)$ (Fig. 2c) y variación Dap de las especies arbóreas $\left(\mathrm{r}^{2}=0.31 ; \mathrm{P}<0.001\right)$ (Fig. 2d). La diversidad de Shannon presentó una relación significativa con el CBS pero menos fuerte que las demás variables $\left(\mathrm{r}^{2}=0.20 ; \mathrm{P}<0.01\right)$.

La relación del CBS con la edad de los sistemas (Fig. 2b) tiene la forma de una curva de saturación, lo que indica una acumulación de biomasa inicial muy rápida, seguida de una desaceleración más adelante en la sucesión. En todos los sistemas agroforestales evaluados, la acumulación de la biomasa se incrementa con la edad, debido al aumento de la abundancia de especies con mayor biomasa individual (Fig. 3) y mayor variación en altura y Dap (Fig. 4). El potencial de acumulación de biomasa en sistemas agroforestales como el café sombreado con 20 a 30 años de edad $(69.9 \pm 6.9 \mathrm{Mg} / \mathrm{ha})$ es similar al bosque secundario de 40 a 60 años de edad (58.1 $\pm 7.6 \mathrm{Mg} /$ ha; Fig. 2b).

Partición de la varianza: La variación explicada para el CBS en su conjunto representó el $85.74 \%$ (Fig. 5). La mayor proporción se debió al efecto compartido entre la riqueza y la estructura (42.69\%). Entretanto el mayor efecto puro sobre el CBS se relacionó con la riqueza $(12.67 \%)$, seguido por la estructura
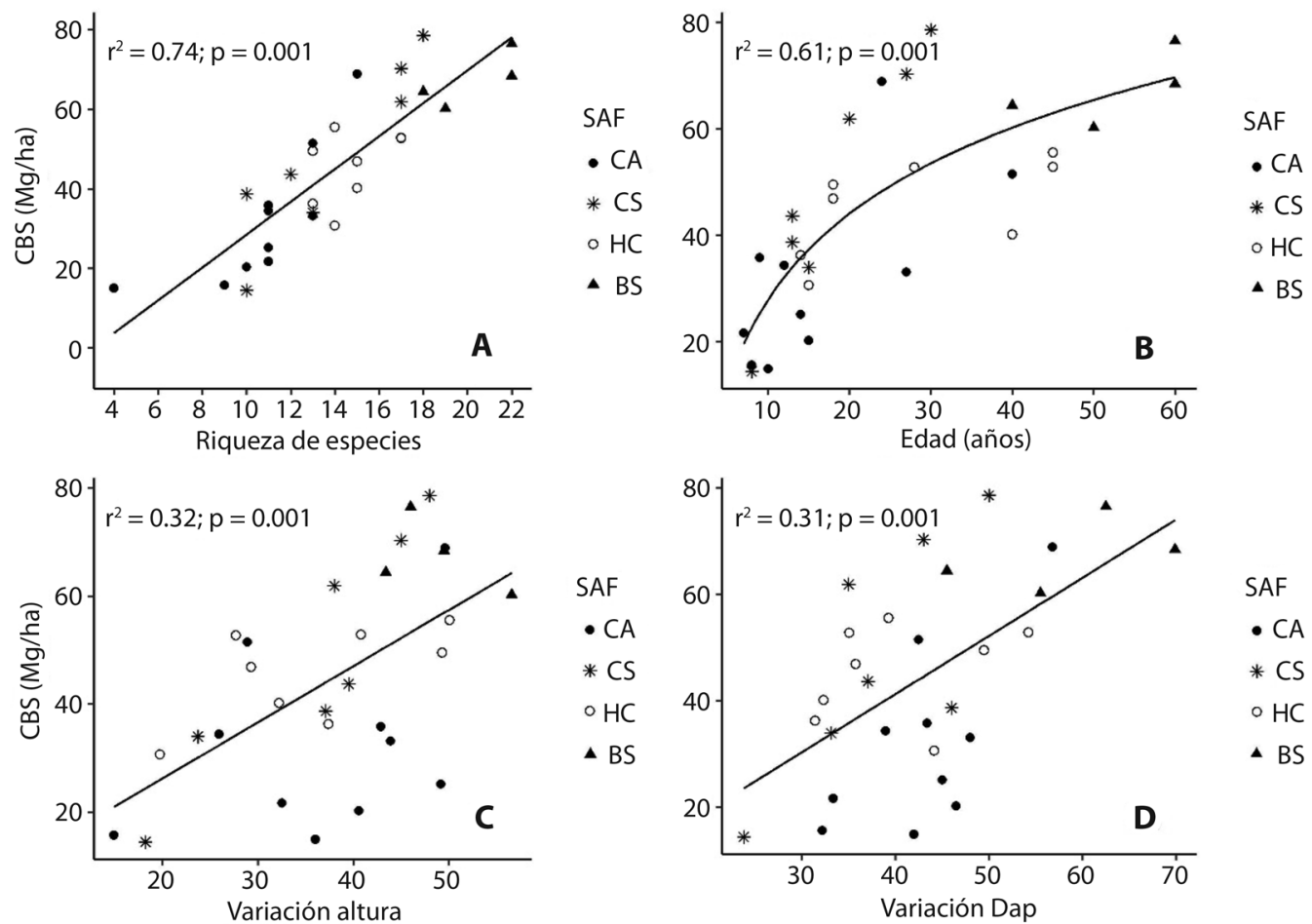

Fig. 2. Relación entre el carbono de la biomasa sobre el suelo (CBS) y la riqueza de especies (a), edad (b), variación altura (c) y variación Dap (d), en sistemas agroforestales (cacao agroforestal/CA, café sombreado/CS, huerto casero/HC) y en bosque secundario (BS) en la Amazonía Sur, Bolivia.

Fig. 2. Relationship between the carbon of the aboveground biomass (AGB) and species richness (a), age (b), height variation (c) and DBH variation (d), in agroforestry systems (cocoa agroforestry/CA, shaded coffee/CS, homegarden/HC), and secondary forest (BS) in the Southern Amazon, Bolivia. 


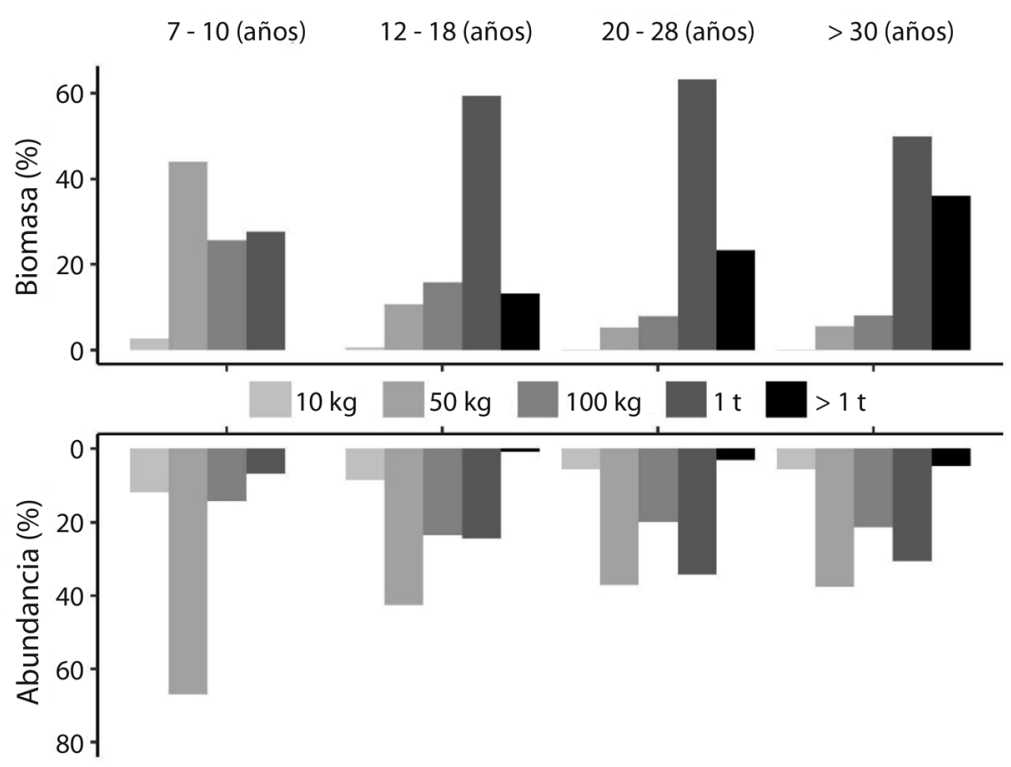

Fig. 3. Distribución porcentual de la biomasa y la abundancia de árboles con diferente biomasa individual (kg) en sistemas agroforestales con diferentes edades en la Amazonía Sur, Bolivia.

Fig. 3. Percentage distribution of biomass and abundance of trees with different individual biomass $(\mathrm{kg})$ in agroforestry systems with different ages in the Southern Amazon, Bolivia.

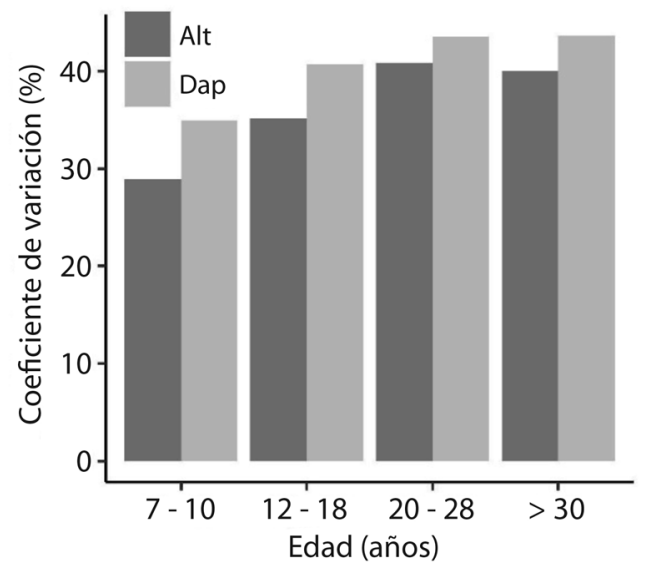

Fig. 4. Distribución del coeficiente de variación (\%) de altura y Dap de especies arbóreas en sistemas agroforestales con diferentes edades en la Amazonía Sur, Bolivia.

Fig. 4. Distribution of variation coefficient (\%) of height and $\mathrm{DBH}$ of tree species in agroforestry systems with different ages in the Southern Amazon, Bolivia.

$(8.76 \%)$ y variación de la estructura (4.77 $\%)$. El efecto puro de las tres variables en el modelo fue significativo. Por su parte, el efecto compartido por las tres fracciones riqueza, estructura y variación de la estructura fue 13.18 $\%$, y en menor proporción los efectos combinados riqueza y variación de la estructura $(0 \%)$, estructura y variación de la estructura (4.72\%).

\section{DISCUSIÓN}

El estudio evidenció relaciones significativas entre la riqueza de especies y el carbono de la biomasa aérea en sistemas agroforestales (SAF) tropicales, relación que también es encontrada en ecosistemas naturales (Poorter et al., 2015; Wang, Lei, Ma, Kneeshaw, \& Peng, 2011; Zhang \& Chen, 2015). Un estudio similar en huertos caseros de zonas secas, sugiere que la biomasa aérea es promovida por la riqueza de especies y la variación intraespecífica e interespecífica del tamaño de los árboles (Ali \& Mattsson, 2017b).

En este estudio se observó diferentes sistemas agroforestales, con diferentes edades, riqueza y estructura en la vegetación, además de bosques secundarios avanzados como ecosistemas de referencia. Los sistemas 


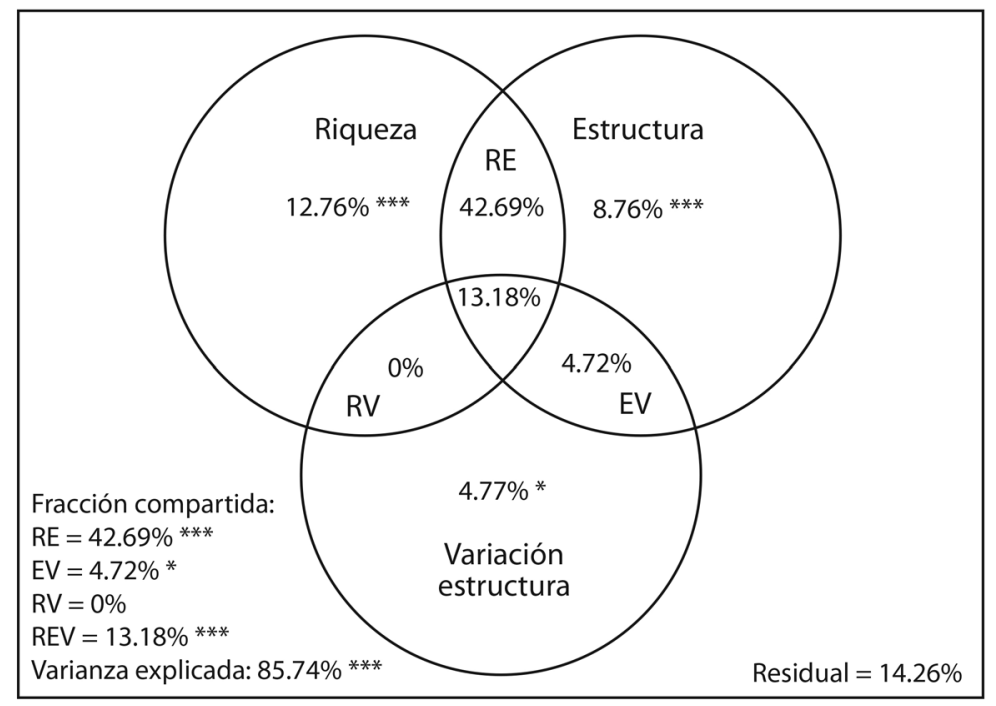

Fig. 5. Partición de varianza para el carbono en la biomasa sobre el suelo (CBS) en sistemas agroforestales con diferentes edades, en la Amazonía Sur, Bolivia. La variación es explicada por las variables, efectos puros: riqueza, estructura y variación estructura; efectos compartidos: riqueza y estructura (RE), estructura y variación estructura (EV), riqueza y variación estructura (RV), riqueza, estructura y variación estructura (REV). Residual es la fracción no explicada.

Fig. 5. Partition of variance for carbon of the biomass aboveground (AGB) in agroforestry systems with different ages, in the Southern Amazon, Bolivia. The variation is explained by the variables, pure effects: richness, structure and structure variation; Shared effects: richness and structure (RE), structure and structure variation (EV), richness and structure variation $(\mathrm{RV})$, richness, structure and structure variation (REV). Residual is the unexplained fraction.

agroforestales más antiguos presentaron una diversidad y riqueza de especies, próximo a los bosques secundarios (Fig. 2a). La diversidad es clave para el funcionamiento y estabilidad de los ecosistemas (Cardinale et al., 2012; Hooper et al., 2012), como también importante para la producción de biomasa (Reich et al., 2012), además la diversidad promueve mayor variedad de rasgos funcionales, patrones de crecimiento y tamaño de las especies (Poorter et al., 2016).

Los resultados resaltan, que los SAF, en particular el café sombreado con 20 a 30 años, pueden acumular cantidades similares de biomasa a los bosques secundarios de 40 a 60 años de edad (Fig. 2b). Una explicación para esa relación de biomasa entre estos sistemas es el manejo, en el sistema café sombreado, las especies arbóreas son seleccionadas, el sistema continúa siendo enriquecido con nuevas especies y con la poda mantienen una alta variación en la altura y Dap (Fig. 2c, Fig. 2d), permitiendo una utilización más eficiente de los recursos (Brassard et al., 2013), resultando en mayor producción de biomasa, considerando que en estos sistemas los agricultores no adicionan fertilizantes. Caso contrario, los bosques secundarios ricos en especies, continúan siendo perturbados por la extracción de madera, afectando la estructura de la vegetación y con esto su efecto negativo en las reservas de biomasa aérea.

Los resultados de esta investigación concuerdan con la idea de que la estratificación de la vegetación puede regular la diversidad de especies (Reich et al., 2012; Zhang, Chen, \& Reich, 2012; Zhang \& Chen, 2015), permitiendo una utilización más eficiente de los recursos luz, agua y nutrientes (Poorter et al., 2015; Ali, Yan, Chang, Cheng, \& Liu, 2017), mejorando la producción de biomasa aérea debido al efecto de complementariedad de nichos (Loreau, 2000; Díaz \& Cabido, 2001; Coome, Kunstler, Canham, \& Wright, 2009; 
Brassard et al., 2013). Por lo tanto, sistemas simples que no consideren el efecto positivo del espacio vertical y horizontal como factor regulador de la diversidad y aprovechamiento de los recursos, pueden disminuir su capacidad de producción de biomasa (Reich et al., 2012; Poorter et al., 2016). Esta relación fue encontrada en monocultivos de cacao y sistemas simples de café, con menor capacidad de producción de biomasa, comparados con sistemas multiestratos y biodiversos (Jacobi et al., 2014; De Beenhouwer et al., 2016; Rajab, Leuschner, Barus, Tjoa, \& Hertel, 2016).

La descripción de acumulación de biomasa aérea a lo largo de los años en los sistemas agroforestales y su aproximación en las reservas de biomasa a los bosques secundarios puede ser empleada como una medida de resiliencia (Gunderson, 2000; Gehring, Denich, \& Vlek, 2005) para estos agroecosistemas, considerando que los bosques secundarios reflejan la regeneración natural en la región. En ese contexto los SAF pueden desempeñar un rol importante, como reservorios de carbono, corredores biológicos y refugio para la biodiversidad (Faria, Paciencia, Dixo, Laps, \& Baumgarten, 2007; Caudill, DeClerck, \& Husband, 2015; Zomer et al., 2016).

La acumulación del carbono en la biomasa en el tiempo, tomó la forma de una curva de saturación con la edad de los sistemas (Fig. 2b). Esto sugiere que el carbono en la biomasa, está relacionada con el crecimiento de las especies arbóreas en el tiempo (Schroth et al., 2015), un incremento de biomasa inicial rápido y una desaceleración más adelante. Por eso, un análisis de partición de la varianza donde incluimos a la edad como variable explicativa, encontramos que la edad como factor puro no tiene un efecto directo sobre el carbono de la biomasa (Apéndice 3). Por otra parte, la biomasa aérea en los SAF es dinámico, sujeto a factores de manejo y perturbaciones (poda, selección de especies, sequias, entre otros) que afectan la producción, almacenamiento y perdida de biomasa.

La partición de la varianza (Fig. 5) sugiere que la riqueza, la estructura y variación de la estructura (altura y Dap) de las especies arbóreas son los principales mecanismos para causar efectos positivos sobre el carbono en la biomasa en los sistemas agroforestales. Estas relaciones probablemente resultaron por la distribución de la vegetación en los diferentes estratos, que se da a través del aumento en la desigualdad en la altura y Dap de las especies arbóreas en el tiempo (Fig. 4), lo que significa una ocupación más completa de los nichos por encima y por debajo del suelo, resultando en mayor capacidad de aprovechamiento de los recursos, luz, agua y nutrientes (Brassard et al., 2013; Ali \& Mattsson, 2017b), para ser transformados en biomasa a través de la fotosíntesis (Mercado et al., 2011; Asner et al., 2014). Estos resultados agregan un nuevo valor en el entendimiento de los efectos de la riqueza sobre la biomasa aérea, y el funcionamiento en los sistemas agroforestales. En ese contexto los SAF tienen una participación importante en el potencial de almacenamiento de carbono, como también en la conservación de la biodiversidad y mayor capacidad de resiliencia.

Por lo tanto, la conversión de bosques naturales en paisajes de producción agrícola debe considerar la práctica de sistemas agroforestales, ricos en especies, sobre todo con especies de uso múltiple y con el mantenimiento de la estratificación del sistema, por ser más eficiente en el uso de los recursos, además de proporcionar mayores beneficios y servicios a los medios de vida de los agricultores, también pueden ser más resilientes a los impactos del cambio climático. Para eso, el desarrollo de políticas públicas para la promoción de los sistemas agroforestales y asistencia técnica son fundamentales.

\section{AGRADECIMIENTOS}

A la Coordinación de Perfeccionamiento de Personal de Nivel Superior (CAPES) por la beca concedida. Al Centro de Investigación Agrícola Tropical (CIAT) y al Centro de Investigación y Promoción del Campesinado (CIPCA) por el apoyo logístico y contacto con los agricultores. A Grober Barja y Julio Cesar 
Llanos por el apoyo en campo, a Yner Juárez y a todos los agricultores que fueron parte de este trabajo de investigación.

\section{RESUMEN}

Los sistemas agroforestales diversos concilian la producción de alimentos, la conservación de la biodiversidad y la provisión de servicios ecosistémicos como el secuestro de carbono atmosférico. Sin embargo, el papel de la riqueza florística sobre la producción de carbono en la biomasa de estos sistemas no está claro. Este estudio evaluó el efecto de la riqueza de especies y la estructura de la vegetación sobre el carbono en la biomasa de diferentes sistemas agroforestales, en la Amazonía Sur de Bolivia. Para eso, fueron estudiados 25 sistemas agroforestales y 4 bosques secundarios, en los departamentos de Santa Cruz y Beni. En cada sistema se instalaron parcelas circulares de $1963 \mathrm{~m}^{2}$, donde la vegetación (árboles, arbustos y herbáceas) y necromasa (hojarasca, ramas y árboles muertos) fueron muestreados. Se utilizó funciones lineales y logarítmicas para evaluar el efecto de la riqueza y estructura de la vegetación sobre el carbono; y la partición de la varianza para examinar el efecto puro y compartido de las variables riqueza y estructura. Las regresiones mostraron una relación positiva fuerte de la riqueza de especies sobre el carbono de la biomasa $\left(\mathrm{r}^{2}=\right.$ 0.74; $\mathrm{P}<0.001$ ). En la partición de la varianza, el $85.7 \%$ de la variabilidad del carbono fue explicada por la riqueza, estructura y variación de la estructura. De forma aislada, la riqueza explicó el $12.7 \%$, la estructura el $8.8 \%$ y la variación de la estructura el $4.8 \%$. Estos resultados confirman que el carbono en la biomasa sobre el suelo aumenta con la riqueza de especies y la variación estructural de la vegetación. Por lo tanto, sistemas agroforestales más biodiversos y estratificados son más eficientes en el uso de los recursos y pueden contribuir con la mitigación del cambio climático.

Palabras clave: biodiversidad; biomasa; fórmula alométrica; Amazonía; mitigación del cambio climático.

\section{REFERENCIAS}

Albrecht, A., \& Kandji, S. T. (2003). Carbon sequestration in tropical agroforestry systems. Agriculture, Ecosystems and Environment, 99, 15-27. DOI: 10.1016/ S0167-8809(03)00138-5

Ali, A., \& Mattsson, E. (2017a). Individual tree size inequality enhances aboveground biomass in homegarden agroforestry systems in the dry zone of Sri Lanka. Science of the Total Environment, 575, 6-11. DOI: $10.1016 /$ j.scitotenv.2016.10.022

Ali, A., \& Mattsson, E. (2017b). Disentangling the effects of species diversity, and intraspecific and interspecific tree size variation on aboveground biomass in dry zone homegarden agroforestry systems. Science of the Total Environment, 598, 38-48. DOI: 10.1016/j. scitotenv.2017.04.131

Ali, A., Yan, E. R., Chang, S. X., Cheng, J. Y., \& Liu, X. Y. (2017). Community-weighted mean of leaf traits and divergence of wood traits predict aboveground biomass in secondary subtropical forests. Science of the Total Environment, 574, 654-662. DOI:10.1016/j. scitotenv.2016.09.022

Andrade, H., Segura, M., Somarriba, E., \& Villalobos, M. (2008). Valoración biofísica y financiera de la fijación de carbono por uso del suelo en fincas cacaoteras indígenas de Talamanca, Costa Rica. Agroforestería en Las Américas, 46, 45-50.

Asner, G. P., Martin, R. E., Tupayachi, R., Anderson, C. B., Sinca, F., Carranza-Jiménez, L., \& Martínez, P. (2014). Amazonian functional diversity from forest canopy chemical assembly. Proceedings of the National Academy of Sciences, 111(15), 5604-5609. DOI:10.1073/pnas.1401181111

Atangana, A., Khasa, D., Chang, S., \& Degrande, A. (2014). Major Agroforestry Systems of the Humid Tropics. En A. Atangana, D. Khasa, S. Chang, \& A. Degrande (Eds.), Tropical Agroforestry (pp. 49-93). Dordrecht, Holanda: Springer.

Borcard, D., Gillet, F., \& Legendre, P. (2011). Numerical Ecology with R. New York, USA: Springer-Verlag.

Brassard, B. W., Chen, H. Y. H., Cavard, X., Laganière, J., Reich, P. B., Bergeron, Y., ... Yuan, Z. (2013). Tree species diversity increases fine root productivity through increased soil volume filling. Journal of Ecology, 101(1), 210-219. DOI:10.1111/1365-2745.12023

Brown, S. (1997). Estimating biomass and biomass change of tropical forests: a primer. Roma, Italia: FAO.

Brown, S. (2002). Measuring, monitoring, and verification of carbon benefits for forest-based projects. Philosophical Transactions of the Royal Society, 360, 16691683. DOI:10.1098/rsta.2002.1026

Cardinale, B. J., Duffy, J. E., González, A., Hooper, D. U., Perrings, C., Venail, P., ... Naeem, S. (2012). Biodiversity loss and its impact on humanity. Nature, 486(7401), 59-67. DOI:10.1038/nature 11148

Cardinale, B. J., Matulich, K. L., Hooper, D. U., Byrnes, J. E., Duffy, E., Gamfeldt, L., ... Gonzalez, A. (2011). The functional role of producer diversity in ecosystems. American Journal of Botany, 98(3), 572-592. DOI:10.3732/ajb.1000364

Cardozo, E. G., Muchavisoy, H. M., Silva, H. R., Zelarayán, M. L. C., Leite, M. F. A., Rousseau, G. X., \& Gehring, C. (2015). Species richness increases income in agroforestry systems of eastern Amazonia. Agroforestry Systems, 89(5), 901-916. DOI:10.1007/ s10457-015-9823-9 
Caudill, S. A., De Clerck, F. J. A., \& Husband, T. P. (2015). Connecting sustainable agriculture and wildlife conservation: Does shade coffee provide habitat for mammals? Agriculture, Ecosystems and Environment, 199, 85-93. DOI:10.1016/j.agee.2014.08.023

Chave, J., Andalo, C., Brown, S., Cairns, M. A., Chambers, J. Q., Eamus, D., ... Yamakura, T. (2005). Tree allometry and improved estimation of carbon stocks $\backslash$ rand balance in tropical forests. Oecología, 145, 87-99.

Chave, J., Réjou-Méchain, M., Búrquez, A., Chidumayo, E., Colgan, M. S., Delitti, W., ... Vieilledent, G. (2014). Improved allometric models to estimate the aboveground biomass of tropical trees. Global Change Biology, 20(10), 3177-3190. DOI:10.1111/ gcb. 12629

Chisholm, R. A., Muller-Landau, H. C., Abdul Rahman, K., Bebber, D. P., Bin, Y., Bohlman, S. A., ... Zimmerman, J. K. (2013). Scale-dependent relationships between tree species richness and ecosystem function in forests. Journal of Ecology, 101(5), 1214-1224. DOI:10.1111/1365-2745.12132

Clark, J. S., Dietze, M., Chakraborty, S., Agarwal, P. K., Ibanez, I., LaDeau, S., \& Wolosin, M. (2007). Resolving the biodiversity paradox. Ecology Letters, 10(8), 647-659. DOI:10.1111/j.1461-0248.2007.01041.x

Coomes, D. A., Allen, R. B., Scott, N. A., Goulding, C., \& Beets, P. (2002). Designing systems to monitor carbon stocks in forests and shrublands. Forest Ecology and Management, 164(1-3), 89-108. DOI:10.1016/ S0378-1127(01)00592-8

Coomes, D. A., Kunstler, G., Canham, C. D., \& Wright, E. (2009). A greater range of shadetolerance niches in nutrient-rich forests: an explanation for positive richness-productivity relationships? Journal of Ecology, 97(4), 705-717. DOI:10.1111/j.1365-2745.2009.01507.x

Davidson, E. A., de Araújo, A. C., Artaxo, P., Balch, J. K., Brown, I. F., C. Bustamante, M. M., ... Wofsy, S. C. (2012). The Amazon basin in transition. Nature, 481(7381), 321-328. DOI:10.1038/nature10717

De Beenhouwer, M., Geeraert, L., Mertens, J., Van Geel, M., Aerts, R., Vanderhaegen, K., \& Honnay, O. (2016). Biodiversity and carbon storage co-benefits of coffee agroforestry across a gradient of increasing management intensity in the SW Ethiopian highlands. Agriculture, Ecosystems and Environment, 222, 193199. DOI:10.1016/j.agee.2016.02.017

Díaz, S., \& Cabido, M. (2001). Vive la différence: plant functional diversity matters to ecosystem processes. Trends in Ecology \& Evolution, 16(11), 646-655. DOI:10.1016/S0169-5347(01)02283-2

Faria, D., Paciencia, M. L. B., Dixo, M., Laps, R. R., \& Baumgarten, J. (2007). Ferns, frogs, lizards, birds and bats in forest fragments and shade cacao plantations in two contrasting landscapes in the Atlantic forest, Brazil. Biodiversity and Conservation, 16(8), 23352357. DOI:10.1007/s10531-007-9189-Z

Fauset, S., Johnson, M. O., Gloor, M., Baker, T. R., Monteagudo, M., A., Brienen, R. J. W., ... Phillips, O. L. (2015). Hyperdominance in Amazonian forest carbon cycling. Nature Communications, 6, 6857. DOI: $10.1038 /$ ncomms 7857

Flombaum, P., \& Sala, O. E. (2008). Higher effect of plant species diversity on productivity in natural than artificial ecosystems. Proceedings of the National Academy of Sciences of the United States of America, 105(16), 6087-6090. DOI:10.1073/pnas.0704801105

Gamfeldt, L., Snall, T., Bagchi, R., Jonsson, M., Gustafsson, L., Kjellander, P., ... Bengtsson, J. (2013). Higher levels of multiple ecosystem services are found in forests with more tree species. Nature Communications, 4, 1340. DOI:10.1038/ncomms2328

Gehring, C., Denich, M., \& Vlek, P. L. G. (2005). Resilience of secondary forest regrowth after slashand-burn agriculture in central Amazonia. Journal of Tropical Ecology, 21(5), 519-527. DOI:10.1017/ S0266467405002543

Gehring, C., Park, S., \& Denich, M. (2008). Close relationship between diameters at $30 \mathrm{~cm}$ height and at breast height (DBH). Acta Amazonica, 38(1), 71-76. DOI: $10.1590 /$ S0044-59672008000100008

Gunderson, L. H. (2000). Ecological Resilience-In Theory and Application. Annual Review of Ecology and Systematics, 31(1), 425-439. DOI:10.1146/annurev. ecolsys.31.1.425

Hooper, D. U., Adair, E. C., Cardinale, B. J., Byrnes, J. E. K., Hungate, B. A., Matulich, K. L., ... Connor, M. I. (2012). A global synthesis reveals biodiversity loss as a major driver of ecosystem change. Nature, 486(7401), 105-108. DOI:10.1038/nature11118

IPCC. (2000). Land use, land-use change, and forestry. Cambridge, UK: Cambridge University Press.

IPCC. (2003). Good Practice Guidance for Land Use, Land-Use Change and Forestry. IPCC Guidelines for National Greenhouse Gas Inventories. Cambridge, UK: Cambridge University Press.

IPCC. (2007). Climate change 2007: the physical science basis. Contribution of Working Group I to the Fourth Assessment Report of the Intergovernmental Panel on Climate Change. Cambridge, UK: Cambridge University Press.

Jacobi, J., Andres, C., Schneider, M., Pillco, M., Calizaya, P., \& Rist, S. (2014). Carbon stocks, tree diversity, and the role of organic certification in different cocoa production systems in Alto Beni, Bolivia. Agroforestry Systems, 88(6), 1117-1132. DOI:10.1007/ s10457-013-9643-8 
Justiniano, M. J., Peña-Claros, M., Toledo, M., Jordán, C., Vargas, I., Gutiérrez, M., \& Montero, J. C. (2004). Guía Dendrológica de Especies Forestales de Bolivia (Vol. 2). Santa Cruz, Bolivia: BOLFOR.

Kumar, B. M. (2006). Carbon sequestration potential of tropical homegardens. In B. M. Kumar \& P. K. R. Nair (Eds.), Tropical Homegardens (pp. 185-204). Dordrecht, Holanda: Springer.

Kumar, B. M., \& Nair, P. K. R. (2004). The enigma of tropical homegardens. Agroforestry System, 61(1-3), 135152. DOI:10.1023/B:AGFO.0000028995.13227.ca

Leite, M. F. A., Luz, R. L., Muchavisoy, K. H. M., Zelarayán, M. L. C., Cardoso, E. G., Moraes, F. H. R., ... Gehring, C. (2016). The effect of land use on aboveground biomass and soil quality indicators in spontaneous forests and agroforests of eastern Amazonia. Agroforestry Systems, 90(6), 1009-1023. DOI:10.1007/s10457-015-9880-0

Loreau, M. (2000). Biodiversity and ecosystem functioning: recent theoretical advances. Oikos, 91(1), 3-17. DOI:10.1034/j.1600-0706.2000.910101.x

Loreau, M., Naeem, S., Inchausti, P., Bengtsson, J., Grime, J. P., Hector, A., ... Wardle, D. A. (2001). Ecology: Biodiversity and ecosystem functioning: Current knowledge and future challenges. Science, 294(5543), 804-808. DOI:10.1126/science. 1064088

Matocha, J., Schroth, G., Hills, T., \& Hole, D. (2012). Integrating Climate Change Adaptation and Mitigation through Agroforestry and Ecosystem Conservation. In P. Nair \& D. Garrity (Eds.), Agroforestry - The Future of Global Land Use (pp. 105-126). Dordrecht, Holanda: Springer.

Mercado, L. M., Patino, S., Domingues, T. F., Fyllas, N. M., Weedon, G. P., Sitch, S., ... Lloyd, J. (2011). Variations in Amazon forest productivity correlated with foliar nutrients and modelled rates of photosynthetic carbon supply. Philosophical Transactions of the Royal Society, 366(1582), 3316-3329. DOI:10.1098/rstb.2011.0045

Müller, R., Müller, D., Schierhorn, F., Gerold, G., \& Pacheco, P. (2012). Proximate causes of deforestation in the Bolivian lowlands: An analysis of spatial dynamics. Regional Environmental Change, 12(3), 445-459. DOI:10.1007/s10113-011-0259-0

Nair, P. K. R. (1993). An Introduction to Agroforestry. Londres, Reino Unido: Kluwer Academic Publishers.

Nair, P. K. R. (2014). Agroforestry: Practices and Systems. Encyclopedia of Agriculture and Food Systems, 1, 270282. DOI:10.1016/B978-0-444-52512-3.00021-8

Nair, P. K. R., Kumar, B. M., \& Nair, V. D. (2009). Agroforestry as a strategy for carbon sequestration. Journal of Plant Nutrition and Soil Science, 172(1), 10-23. DOI:10.1002/jpln.200800030
Nelson, B. W., Mesquita, R., Pereira, J. L. G., García Aquino De Souza, S., Teixeira Batista, G., \& Bovino Couto, L. (1999). Allometric regressions for improved estimate of secondary forest biomass in the central Amazon. Forest Ecology and Management, 117(1-3), 149-167. DOI:10.1016/S0378-1127(98)00475-7

Nogueira, E. M., Fearnside, P. M., \& Nelson, B. W. (2008). Normalization of wood density in biomass estimates of Amazon forests. Forest Ecology and Management, 256(5), 990-996. DOI:10.1016/j.foreco.2008.06.001

Oksanen, J., Blanchet, F. G., Kindt, R., Legendre, P., Minchin, P. R., O’Hara, R. B., ... Wagner, H. (2015). Vegan: Community Ecology Package. $R$ Package Version 2.3-1. DOI:10.4135/9781412971874.n145

Pearson, T., Walker, S., \& Brown, S. (2005). Sourcebook for land use, land-use change and forestry projects. Nueva York, EUA: World Bank.

Peel, M. C., Finlayson, B. L., \& McMahon, T. A. (2007). Updated world map of the Köppen-Geiger climate classification. Hydrology and Earth System Sciences, 11(5), 1633-1644. DOI:10.5194/hess-11-1633-2007

Pinho, R. C., Miller, R. P., \& Alfaia, S. S. (2012). Agroforestry and the Improvement of Soil Fertility: A View from Amazonia. Applied and Environmental Soil Science, 2012, 1-11. DOI:10.1155/2012/616383

Poorter, L., Bongers, F., Aide, T. M., Almeyda Zambrano, A. M., Balvanera, P., Becknell, J. M., ... Rozendaal, D. M. A. (2016). Biomass resilience of Neotropical secondary forests. Nature, 530(7589), 211-214. DOI:10.1038/nature16512

Poorter, L., van der Sande, M. T., Thompson, J., Arets, E. J. M. M., Alarcón, A., Álvarez-Sánchez, J., ... PeñaClaros, M. (2015). Diversity enhances carbon storage in tropical forests. Global Ecology and Biogeography, 24(11), 1314-1328. DOI:10.1111/geb.12364

Quesada, C. A., Lloyd, J., Anderson, L. O., Fyllas, N. M., Schwarz, M., \& Czimczik, C. I. (2011). Soils of Amazonia with particular reference to the RAINFOR sites. Biogeosciences, 8(6), 1415-1440. DOI:10.5194/ bg-8-1415-2011

Quesada, C. A., Phillips, O. L., Schwarz, M., Czimczik, C. I., Baker, T. R., Patiño, S., \& Lloyd, J. (2012). Basin-wide variations in Amazon forest structure and function are mediated by both soils and climate. Biogeosciences, 9(6), 2203-2246. DOI:10.5194/ bg-9-2203-2012

R Core Team. (2016). R Development Core Team. R: $A$ Language and Environment for Statistical Computing. Retrieved from https://www.r-project.org

Rajab, Y. A., Leuschner, C., Barus, H., Tjoa, A., \& Hertel, D. (2016). Cacao cultivation under diverse shade tree cover allows high carbon storage and sequestration 
without yield losses. PLOS ONE, 11(2). DOI:10.1371/ journal.pone.0149949

Reich, P. B., Tilman, D., Isbell, F., Mueller, K., Hobbie, S. E., Flynn, D. F. B., \& Eisenhauer, N. (2012). Impacts of biodiversity loss escalate through time as redundancy fades. Science, 336(6081), 589-592. DOI:10.1126/science.1217909

Rousseau, G. X., Rioux, S., \& Dostaler, D. (2006). Partitioning the spatial and environmental variation of Sclerotinia stem rot on soybean. Soil Biology and Biochemistry, 38(12), 3343-3358. DOI:10.1016/j. soilbio.2006.04.052

Schroth, G., Bede, L. C., Paiva, A. O., Cassano, C. R., Amorim, A. M., Faria, D., ... Lôbo, R. N. (2015). Contribution of agroforests to landscape carbon storage. Mitigation and Adaptation Strategies for Global Change, 20(7), 1175-1190. DOI:10.1007/ s11027-013-9530-7

Schroth, G., D’Angelo, S. A., Teixeira, W. G., Haag, D., \& Lieberei, R. (2002). Conversion of secondary forest into agroforestry and monoculture plantations in Amazonia: Consequences for biomass, litter and soil carbon stocks after 7 years. Forest Ecology and Management, 163(1-3), 131-150. DOI:10.1016/ S0378-1127(01)00537-0

Segura, M., Kanninen, M., \& Suárez, D. (2006). Allometric models for estimating aboveground biomass of shade trees and coffee bushes grown together. Agroforestry Systems, 68(2), 143-150. DOI:10.1007/ s10457-006-9005-x

Van Wagner, C. E. (1968). The line intersect method in forest fuel sampling. Forest Science, 14, 20-26.
Vargas, I., Mostacedo, B., \& Jordán, C. (2005). Guía Ilustrada de las Principales Especies Forestales de Bolivia. Santa Cruz, Bolivia: IBIF,WWF.

Wang, W., Lei, X., Ma, Z., Kneeshaw, D. D., \& Peng, C. (2011). Positive relationship between aboveground carbon stocks and structural diversity in sprucedominated forest stands in New Brunswick, Canada. Forest Science, 57(6), 506-515. DOI:10.1111/ oik.01525

Yamada, M., \& Gholz, H. L. (2002). An evaluation of agroforestry systems as a rural development option for the Brazilian Amazon. Agroforestry Systems, 55(2), 81-87. DOI:10.1023/A:1020523107243

Young, A. (1997). Agroforestry for soil management $\left(2^{\circ}\right.$ ed). Wallingford, Reino Unido: CAB international.

Zanne, A. E., Lopez-Gonzalez, G., Coomes, D. A. A., Ilic, J., Jansen, S., Lewis, S. L. S. L., ... Chave, J. (2009). Global wood density database. Dryad, 235(February), 33. DOI:10.5061/dryad.234

Zhang, Y., \& Chen, H. Y. H. (2015). Individual size inequality links forest diversity and above-ground biomass. Journal of Ecology, 103(5), 1245-1252. DOI:10.1111/1365-2745.12425

Zhang, Y., Chen, H. Y. H., \& Reich, P. B. (2012). Forest productivity increases with evenness, species richness and trait variation: A global metaanalysis. Journal of Ecology, 100(3), 742-749. DOI:10.1111/j.1365-2745.2011.01944.x

Zomer, R. J., Neufeldt, H., Xu, J., Ahrends, A., Bossio, D., Trabucco, A., ... Wang, M. (2016). Global Tree Cover and Biomass Carbon on Agricultural Land: The contribution of agroforestry to global and national carbon budgets. Scientific Reports, 6, 1-12. DOI:10.1038/srep29987 


\section{APÉNDICE 1}

Ubicación de los sistemas agroforestales muestreado en la Amazonía sur, Bolivia

\section{APPENDIX 1}

Location of agroforestry systems sampled in the Southern Amazon, Bolivia

\begin{tabular}{|c|c|c|c|}
\hline Sistema & Municipio & Edad & Coordenadas \\
\hline \multirow{10}{*}{$\begin{array}{l}\text { Cacao agroforestal (CA) } \\
n=10\end{array}$} & \multirow[t]{2}{*}{ Ascensión de Guarayos } & 7 & $15^{\circ} 46^{\prime} 43^{\prime \prime} \mathrm{S}-63^{\circ} 17^{\prime} 57^{\prime \prime} \mathrm{W}$ \\
\hline & & 8 & $15^{\circ} 49^{\prime} 09^{\prime \prime} \mathrm{S}-62^{\circ} 58^{\prime} 06^{\prime \prime} \mathrm{W}$ \\
\hline & Buena Vista & 10 & $17^{\circ} 28^{\prime} 43^{\prime \prime} \mathrm{S}-63^{\circ} 41^{\prime} 09^{\prime \prime} \mathrm{W}$ \\
\hline & \multirow[t]{2}{*}{ San Andrés } & 15 & $14^{\circ} 58^{\prime} 40^{\prime \prime} \mathrm{S}-64^{\circ} 19^{\prime} 08^{\prime \prime} \mathrm{W}$ \\
\hline & & 40 & $14^{\circ} 58^{\prime} 58^{\prime \prime} \mathrm{S}-64^{\circ} 19^{\prime} 07^{\prime \prime} \mathrm{W}$ \\
\hline & \multirow[t]{3}{*}{ San Ignacio de Moxos } & 24 & $14^{\circ} 53^{\prime} 55^{\prime \prime} \mathrm{S}-64^{\circ} 22^{\prime} 27^{\prime \prime} \mathrm{W}$ \\
\hline & & 9 & $14^{\circ} 55^{\prime} 25^{\prime \prime} \mathrm{S}-65^{\circ} 42^{\prime} 05^{\prime \prime} \mathrm{W}$ \\
\hline & & 12 & $15^{\circ} 12^{\prime} 09^{\prime \prime} \mathrm{S}-65^{\circ} 51^{\prime} 34^{\prime \prime} \mathrm{W}$ \\
\hline & \multirow[t]{2}{*}{ San Javier } & 27 & $14^{\circ} 49^{\prime} 09^{\prime \prime} \mathrm{S}-64^{\circ} 19^{\prime} 09^{\prime \prime} \mathrm{W}$ \\
\hline & & 14 & $14^{\circ} 39^{\prime} 07^{\prime \prime} \mathrm{S}-64^{\circ} 19^{\prime} 40^{\prime \prime} \mathrm{W}$ \\
\hline \multirow{7}{*}{$\begin{array}{l}\text { Café sombreado (CS) } \\
n=7\end{array}$} & Ascensión de Guarayos & 8 & $15^{\circ} 56^{\prime} 08^{\prime \prime} \mathrm{S}-63^{\circ} 03^{\prime} 30^{\prime \prime} \mathrm{W}$ \\
\hline & \multirow[t]{3}{*}{ Buena Vista } & 30 & $17^{\circ} 28^{\prime} 06^{\prime \prime} \mathrm{S}-63^{\circ} 41^{\prime} 09^{\prime \prime} \mathrm{W}$ \\
\hline & & 13 & $17^{\circ} 39^{\prime} 28^{\prime \prime} \mathrm{S}-63^{\circ} 34^{\prime} 06^{\prime \prime} \mathrm{W}$ \\
\hline & & 15 & $17^{\circ} 28^{\prime} 28^{\prime \prime} \mathrm{S}-63^{\circ} 41^{\prime} 06^{\prime \prime} \mathrm{W}$ \\
\hline & \multirow[t]{3}{*}{ San Carlos } & 27 & $17^{\circ} 27^{\prime} 50^{\prime \prime} \mathrm{S}-63^{\circ} 49^{\prime} 06^{\prime \prime} \mathrm{W}$ \\
\hline & & 20 & $17^{\circ} 27^{\prime} 22^{\prime \prime} \mathrm{S}-63^{\circ} 50^{\prime} 27^{\prime \prime} \mathrm{W}$ \\
\hline & & 13 & $17^{\circ} 25^{\prime} 07^{\prime \prime} \mathrm{S}-63^{\circ} 49^{\prime} 54^{\prime \prime} \mathrm{W}$ \\
\hline \multirow{8}{*}{$\begin{array}{l}\text { Huerto casero (HC) } \\
\mathrm{n}=8\end{array}$} & Ascensión de Guarayos & 18 & $15^{\circ} 49^{\prime} 09^{\prime \prime} \mathrm{S}-63^{\circ} 00^{\prime} 59^{\prime \prime} \mathrm{W}$ \\
\hline & Buena Vista & 40 & $17^{\circ} 28^{\prime} 14^{\prime \prime} \mathrm{S}-63^{\circ} 41^{\prime} 55^{\prime \prime} \mathrm{W}$ \\
\hline & \multirow[t]{2}{*}{ San Ignacio de Moxos } & 18 & $14^{\circ} 53^{\prime} 08^{\prime \prime} \mathrm{S}-65^{\circ} 22^{\prime} 37^{\prime \prime} \mathrm{W}$ \\
\hline & & 28 & $14^{\circ} 54^{\prime} 09^{\prime \prime} \mathrm{S}-65^{\circ} 20^{\prime} 07^{\prime \prime} \mathrm{W}$ \\
\hline & \multirow[t]{2}{*}{ San Javier } & 15 & $14^{\circ} 40^{\prime} 10^{\prime \prime} \mathrm{S}-64^{\circ} 19^{\prime} 09^{\prime \prime} \mathrm{W}$ \\
\hline & & 45 & $14^{\circ} 39^{\prime} 07^{\prime \prime} \mathrm{S}-64^{\circ} 19^{\prime} 41^{\prime \prime} \mathrm{W}$ \\
\hline & Urubichá & 45 & $15^{\circ} 40^{\prime} 09^{\prime \prime} \mathrm{S}-63^{\circ} 06^{\prime} 43^{\prime \prime} \mathrm{W}$ \\
\hline & Yapacaní & 14 & $17^{\circ} 22^{\prime} 09^{\prime \prime} \mathrm{S}-63^{\circ} 58^{\prime} 06^{\prime \prime} \mathrm{W}$ \\
\hline \multirow{4}{*}{$\begin{array}{l}\text { Bosque secundário (BS) } \\
\mathrm{n}=4\end{array}$} & Buena Vista & 50 & $17^{\circ} 25^{\prime} 08^{\prime \prime} \mathrm{S}-63^{\circ} 39^{\prime} 21^{\prime \prime} \mathrm{W}$ \\
\hline & \multirow[t]{2}{*}{ San Ignacio de Moxos } & 40 & $15^{\circ} 13^{\prime} 58^{\prime \prime} \mathrm{S}-65^{\circ} 50^{\prime} 08^{\prime \prime} \mathrm{W}$ \\
\hline & & 60 & $15^{\circ} 02^{\prime} 26^{\prime \prime} \mathrm{S}-65^{\circ} 39^{\prime} 08^{\prime \prime} \mathrm{W}$ \\
\hline & Yapacaní & 60 & $17^{\circ} 22^{\prime} 07^{\prime \prime} \mathrm{S}-63^{\circ} 58^{\prime} 08^{\prime \prime} \mathrm{W}$ \\
\hline
\end{tabular}




\section{APÉNDICE 2}

Taxonomía y uso de las diez especies arbóreas más abundantes en los sistemas agroforestales muestreado en la Amazonía sur, Bolivia

\section{APPENDIX 2}

Taxonomy and use of the ten most abundant tree species of the agroforestry systems sampled in the Southern Amazon, Bolivia

\begin{tabular}{lllcl}
\multicolumn{1}{c}{$\begin{array}{c}\text { Nombre } \\
\text { común }\end{array}$} & \multicolumn{1}{c}{ Especie } & \multicolumn{1}{c}{ Familia } & $\begin{array}{c}\text { Abundancia } \\
(\%)\end{array}$ & \multicolumn{1}{c}{ Principales usos } \\
Cacao & Theobroma cacao L. & Malvaceae & 33.8 & Alimento \\
Banano & Musa spp. & Musaceae & 5.6 & Alimento, sombra, materia orgánica \\
Motacú & Attalea princeps Mart. & Arecaceae & 4.3 & Sombra, artesanía, material de construcción \\
Toronja & Citrus paradisi Macfad. & Rutaceae & 3.9 & Alimento \\
Pacay & Inga sp. & Fabaceae & 3.6 & Alimento, sombra, materia orgánica, \\
Manga & Mangifera indica L. & Anacardiaceae & 2.3 & Alimento, madera \\
Mandarina & Citrus reticulata Blanco & Rutaceae & 2.3 & Alimento \\
Naranja & Citrus sinensis L. Osbeck & Rutaceae & 2.2 & Alimento \\
Palta & Persea americana Mill. & Lauraceae & 1.9 & Alimento \\
Jorori & Swartzia jorori Harms & Fabaceae & 1.8 & Sombra, madera \\
Otros & & & 38.1 & \\
\hline
\end{tabular}

\section{APÉNDICE 3 / APPENDIX 3}

Partición de varianza para el carbono en la biomasa sobre el suelo (CBS)

Partition of variance for carbon of the biomass aboveground (CBS)

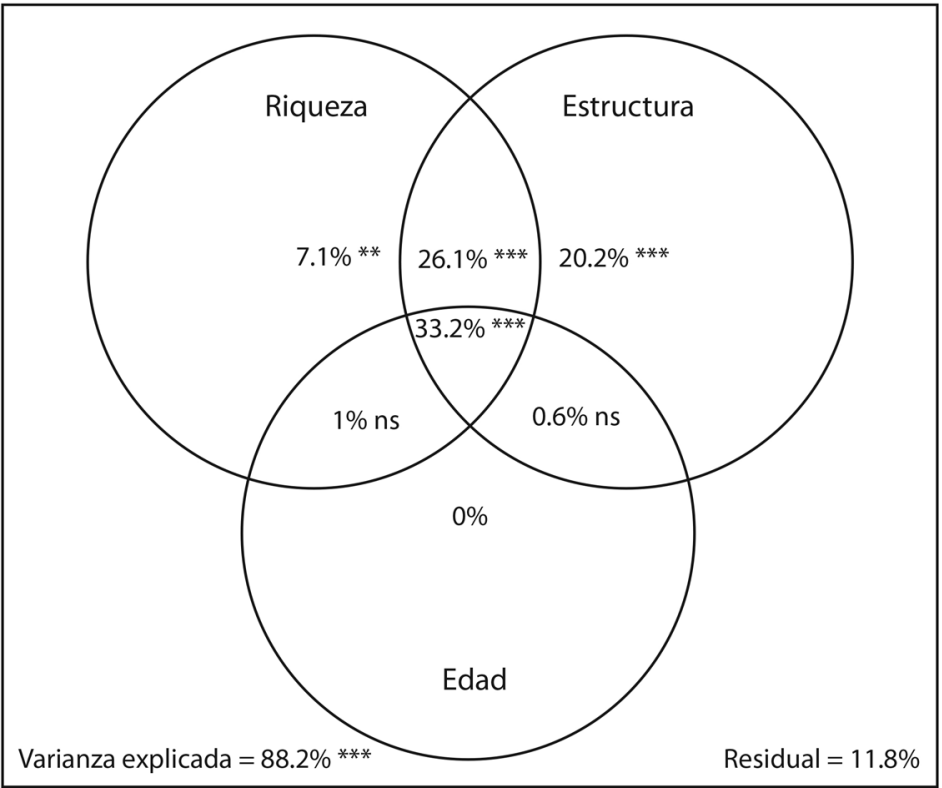

Variables explicativas: riqueza, estructura y edad, en los sistemas agroforestales muestreado en la Amazonía sur, Bolivia. Residual es la fracción no explicada.

Explanatory variables: richness, structure and age, agroforestry systems sampled in the Southern Amazon, Bolivia. Residual is the unexplained fraction. 